\title{
Holographic Interferometry based on photorefractive crystal to measure 3D thermo-elastic distortion of composite structures and comparison with finite element models
}

C. Thizy, F. Eliot, D. Ballhause, K. R. Olympio, R. Kluge, et al.

C. Thizy, F. Eliot, D. Ballhause, K. R. Olympio, R. Kluge, A. Shannon, G. Laduree, D. Logut, M. P. Georges, "Holographic Interferometry based on photorefractive crystal to measure 3D thermo-elastic distortion of composite structures and comparison with finite element models," Proc. SPIE 8788, Optical Measurement Systems for Industrial Inspection VIII, 878807 (13 May 2013); doi: $10.1117 / 12.2020542$ 


\title{
Holographic Interferometry based on photorefractive crystal to measure 3D thermo-elastic distortion of composite structures and comparison with finite element models
}

\author{
C. Thizy ${ }^{*}{ }^{*}$, F. Eliot ${ }^{\mathrm{b}}$, D. Ballhause ${ }^{\mathrm{c}}$, K.R. Olympio ${ }^{\mathrm{c}}$, R. Kluge ${ }^{\mathrm{c}}$, A. Shannon ${ }^{\mathrm{d}}$, G. Laduree $^{\mathrm{d}}$, D. Logut ${ }^{\mathrm{b}}$, \\ M.P. Georges ${ }^{\mathrm{a}}$ \\ ${ }^{a}$ Centre Spatial de Liège - Université de Liège, Avenue du Pré-Aily, 4031 Angleur, Belgium \\ ${ }^{\mathrm{b}}$ EADS-Astrium, 31 Rue des Cosmonautes, 31402 Toulouse, France \\ ${ }^{\mathrm{c}}$ EADS-Astrium, 88039 Friedrichshafen, Germany \\ ${ }^{\mathrm{d}}$ European Space Agency ESTEC, Keplerlaan 1, NL-2200 AG Noordwijk ZH, The Netherlands
}

\begin{abstract}
Thermo-elastic distortions of composite structures have been measured by a holographic camera using a BSO photorefractive crystal as the recording medium. The first test campaign (Phase 1) was performed on CFRP struts with titanium end-fittings glued to the tips of the strut. The samples were placed in a vacuum chamber. The holographic camera was located outside the chamber and configured with two illuminations to measure the relative out-of-plane and in-plane (in one direction) displacements. The second test campaign (Phase 2) was performed on a structure composed of a large Silicon Carbide base plate supported by 3 GFRP struts with glued Titanium end-fittings. Thermo-elastic distortions have been measured with the same holographic camera used in phase 1, but four illuminations, instead of two, have been used to provide the three components of displacement. This technique was specially developed and validated during the phase 2 in CSL laboratory. The system has been designed to measure an object size of typically $250 \times 250$ $\mathrm{mm}^{2}$; the measurement range is such that the sum of the largest relative displacements in the three measurement directions is maximum $20 \mu \mathrm{m}$. The validation of the four-illuminations technique led to measurement uncertainties of $120 \mathrm{~nm}$ for the relative in-plane and out-of-plane displacements, $230 \mathrm{~nm}$ for the absolute in-plane displacement and 400 $\mathrm{nm}$ for the absolute out-of-plane displacement. For both campaigns, the test results have been compared to the predictions obtained by finite element analyses and the correlation of these results was good.
\end{abstract}

Keywords: Holographic interferometry, Photorefractive crystal, Composite structure, 3D, Thermo-elastic distortion, Finite element model,

\section{INTRODUCTION}

Scientific, earth observation and telecommunication spacecrafts are subject to severe thermal environments while their mission performance objectives require always higher stability of the structures to deliver high quality images or to insure accurate pointing. The accurate verification and prediction of the thermo-elastic distortions of these structures are therefore of primary importance for the success of such missions.

Thermo-elastic distortions have first been imposed on the selected hardware by local heating and then measured by a

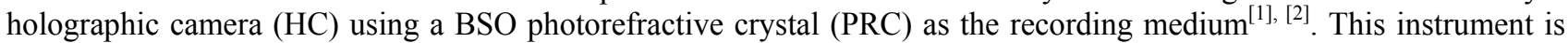
based on real time holographic interferometry (HI). First the hologram of the structure in its initial state is recorded into the PRC. Then a thermal load is applied to the structure, the structure is deformed and the hologram is readout, showing the interference between the transmitted and readout images. In its classical configuration, the HC uses one illumination and measures the relative out-of-plane displacement.

*Cedric.Thizy@ulg.ac.be; phone 0032 43824658; fax 0032 43675613; www.csl.ulg.ac.be

Optical Measurement Systems for Industrial Inspection VIII, edited by Peter H. Lehmann, Wolfgang Osten, Armando Albertazzi, Proc. of SPIE Vol. 8788,

878807 - (c) 2013 SPIE · CCC code: 0277-786X/13/\$18 - doi: 10.1117/12.2020542 
For this study, the HC has been used in two configurations: one with two illuminations to measure the relative out-ofplane (OP) and in-plane (IP) (in one direction) displacements and one with four illuminations to measure the three components of displacement. This latter configuration has been developed and validated for the second phase of the study.

The tests were performed in the frame of an ESA project coordinated by EADS-Astrium who was also in charge of the finite element analysis and the correlation between test and prediction results. The objective of the first phase of this project was to improve and develop analytical predictions and verification of thermo-elastic distortions using sample testing for modeling correlation. The second phase of the project aimed at evaluating and validating the outcomes of phase 1 on a flight representative hardware.

\section{PHOTOREFRACTIVE HOLOGRAPHIC CAMERA}

\subsection{Classical configuration}

The HC is composed of a compact optical head (Figure 1) and an electronic rack containing the $5 \mathrm{~W}$ Nd-YAG laser operating at $532 \mathrm{~nm}$. A single mode optical fiber brings the laser light to the optical head, which makes it a flexible instrument.
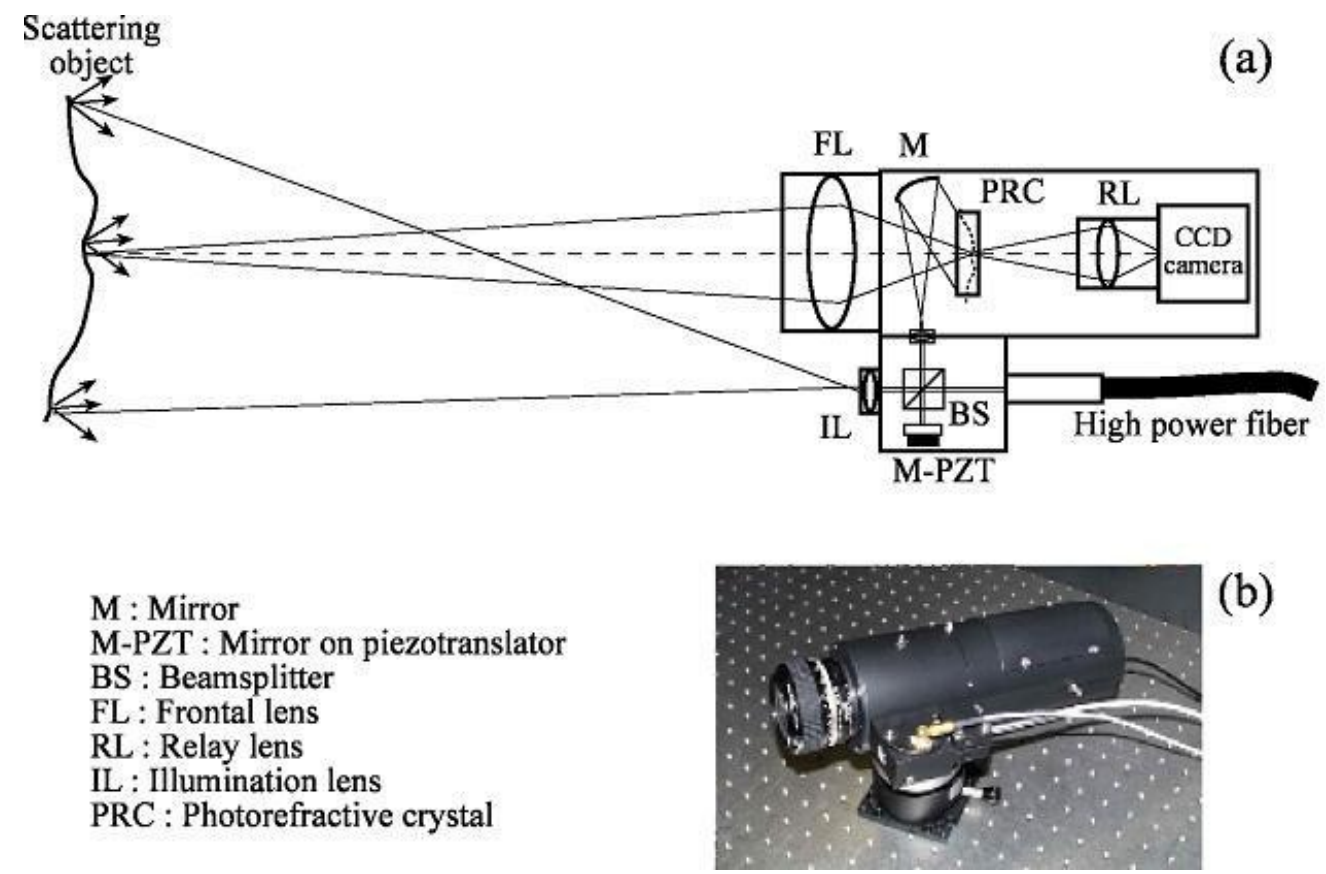

(b)

Figure 1. Holographic camera, (a) scheme principle and (b) picture.

Both recording beams (reference and object) are continuously incident on the crystal. The recording of the hologram takes place under the response time of the photorefractive effect. The latter is mainly driven by the reference beam intensity that can be tuned. The response time to use depends mainly on the external conditions under which the holographic camera is used but generally its value ranges from 5 to 10 seconds if a moderately stable environment is considered (few external vibrations and air turbulences, no need of vibration compensated optical table). This response time being the same at the recording and the readout, it must not be too short in order to allow a proper use of the phaseshifting process during interferograms capture ${ }^{[3],[4]}$. Each time the readout of the hologram is performed, the hologram is partially erased, which means that from the same hologram representing the reference state of the tested object, a limited number of measurements can be performed before the hologram is fully erased (typically four for a response time of $10 \mathrm{~s})$. Once the hologram is erased the instrument is ready for a new hologram recording and measurement. The HC provides high quality fringes and high resolvable fringe density (typically 5pixels/fringe). Relative out-of-plane 
displacements ranging from $20 \mathrm{~nm}$ to $20 \mu \mathrm{m}$ can be measured in one shot. Tested object are usually white powdered to improve laser light scattering.

\subsection{Two-illuminations configuration}

This configuration has been implemented for the measurement of the Poisson coefficient of a polymer sample ${ }^{[5]}$. During the phase 1 of the study the tests have been performed with the samples in a vacuum chamber, the geometry of the two illuminations has therefore been adapted to the design of this chamber (Figure 2).

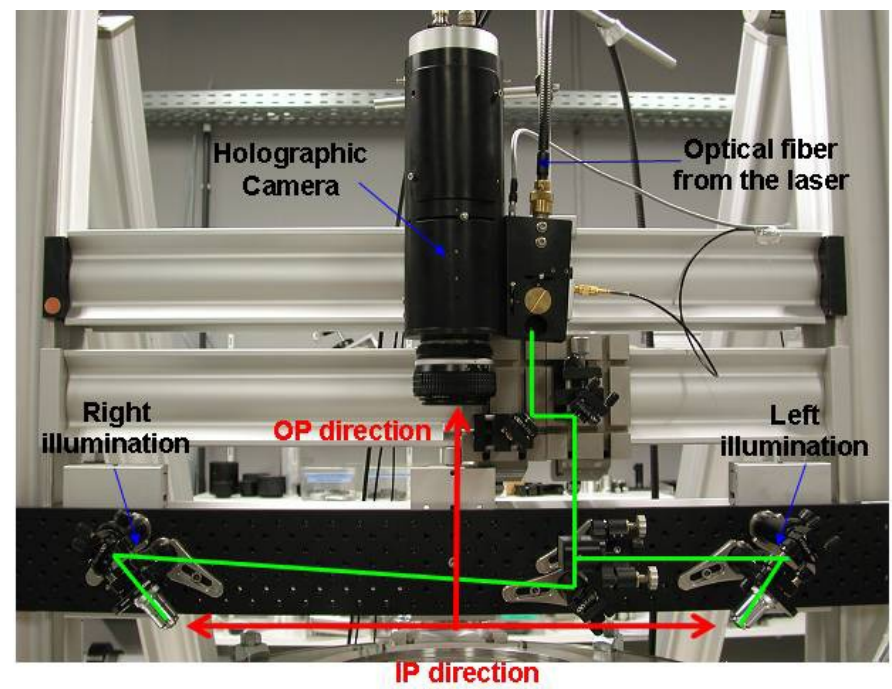

Figure 2. Two-illuminations configuration of the HC.

Two holograms are simultaneously recorded in the crystal, the readout is sequential. In this configuration the relative OP and IP (in one direction) displacements are measured. The combination of two phase maps to obtain these displacements leads to a measurement uncertainty of about $40 \mathrm{~nm}$.

The first tests performed on a sample with a well-known coefficient of thermal expansion (CTE) showed that the measurement of the relative IP displacement was not correct due to the influence of the rigid body translations of the sample. To overcome this issue the solution was to implement a reference sample with a known CTE in the field of view of the $\mathrm{HC}$ and then to scale the results ${ }^{[6]}$.

\subsection{Four-illuminations configuration}

The limitations of the two-illuminations configuration, i.e. only relative displacements measured along two directions and the use of a reference sample to have reliable results, led to the development of a new configuration of the HC. The objective was to measure the three components of the displacement during the tests for the second phase of the study. It has to be noticed that this development was not initially planned in the study, the cost of the development and the hardware had therefore to be as limited as possible.

\subsubsection{Principle}

The HC measures phase difference resulting from the displacement of the surface under test. The general equation for this phase difference $\phi$ at a point $\mathrm{P}$ on the object is:

$$
\phi(P)=\vec{S}(P) \cdot \vec{d}(P)+\phi_{0}
$$

Where $\vec{d}$ is the displacement vector, $\phi_{0}$ is an unknown phase offset (function of rigid body translations and phase unwrapping) and $\vec{S}$ is the sensitivity given by 


$$
\vec{S}(P)=\frac{2 \pi}{\lambda}\left(\overrightarrow{k_{0}}(P)-\overrightarrow{k_{l}}(P)\right)
$$

With $\overrightarrow{k_{0}}$ and $\overrightarrow{k_{l}}$ the observation and illumination vectors, respectively.

For measuring the three components of the displacement, the first solution is to use three illuminations combined with the measurement of the absolute displacement of one point of the tested object or with a zero displacement point on the tested object. The three $\phi_{0}$ are therefore obtained from the absolute measurement of this single point, and the three equations lead to the linear system (equation (3)) that can be solved easily.

$$
\left[\begin{array}{l}
\phi^{1}(P)-\phi_{0}^{1} \\
\phi^{2}(P)-\phi_{0}^{2} \\
\phi^{3}(P)-\phi_{0}^{3}
\end{array}\right]=\left[\begin{array}{lll}
S_{x}^{1}(P) & S_{y}^{1}(P) & S_{z}^{1}(P) \\
S_{x}^{2}(P) & S_{y}^{2}(P) & S_{z}^{2}(P) \\
S_{x}^{3}(P) & S_{y}^{3}(P) & S_{z}^{3}(P)
\end{array}\right]\left[\begin{array}{l}
d_{x}(P) \\
d_{y}(P) \\
d_{z}(P)
\end{array}\right]
$$

With $\phi^{i}(P), \phi_{0}^{i}$ and $\overrightarrow{S^{i}}(P)$ the phase, phase offset and sensitivity vector, respectively, at point $\mathrm{P}$ for the illumination $\mathrm{i}$. In practice, this means that an additional system has to be used to measure the absolute displacement of one point on the object. This makes the experimental set-up more complex and when thermal tests are planned, the thermal impact on this additional system must also be considered.

In order to avoid the additional measurement of the phase offsets, the solution is to use four illuminations instead of three. By using four illuminations and at least the phase data of four points on the object, it is possible to obtain the three components of the displacement. The linear system to solve is then given by ${ }^{[7]}$ :

$$
\left[\begin{array}{l}
{\left[\phi\left(P_{1}\right)\right]} \\
{\left[\phi\left(P_{2}\right)\right]} \\
{\left[\phi\left(P_{3}\right)\right]} \\
{\left[\phi\left(P_{4}\right)\right]}
\end{array}\right]=\left[\begin{array}{ccccc}
{\left[S\left(P_{1}\right)\right]} & 0 & 0 & 0 & I \\
0 & {\left[S\left(P_{2}\right)\right]} & 0 & 0 & I \\
0 & 0 & {\left[S\left(P_{3}\right)\right]} & 0 & I \\
0 & 0 & 0 & {\left[S\left(P_{4}\right)\right] I}
\end{array}\right]\left[\begin{array}{c}
{\left[d\left(P_{1}\right)\right]} \\
{\left[d\left(P_{1}\right)\right]} \\
{\left[d\left(P_{1}\right)\right]} \\
{\left[d\left(P_{1}\right)\right]} \\
{\left[\phi_{0}\right]}
\end{array}\right]
$$

Where $I$ is a fourth rank identity matrix. The sensitivity matrix must not be singular meaning that for each illumination the sensitivity vector must change across the object. Of course more than four object points can be used to possibly have a more reliable and robust solving of the linear system that will then be over-determined.

\subsubsection{Implementation of the technique in the holographic camera}

In order to use the four-illuminations technique with the HC camera, four holograms have to be recorded in the PRC and then readout. The recording/readout of four holograms in a PRC to perform HI has already been studied ${ }^{[8]}$.

To have a more versatile instrument, but also for a budget reason, the design of the four-illuminations module was made such that the instrument could be used with the vacuum chamber used during phase 1 (Figure $5 \mathrm{~b}$ ). The geometry of the four illuminations was therefore imposed by the geometry of the chamber. The maximum object size that can be measured is typically $250 \times 250 \mathrm{~mm}^{2}$ with the object located at about $550 \mathrm{~mm}$ from the illuminations. The diagonal of the square composed of the four illuminations is about $450 \mathrm{~mm}$. It has to be noticed that the four-illuminations module was made of aluminium for cost reason while a low CTE material (such as Invar) would have been more appropriate to limit the relative displacement of the four sources with respect to the tested object.

The four-illuminations module is interfaced on the optical head of the HC and splits the laser beam into four beams using cube beam-splitters and folding mirrors (Figure 3). Four holograms are simultaneously recorded in the PRC; the readout is sequential. In this configuration only one acquisition can be performed from the same reference state of the object. The measurement range is such that the sum of the largest relative displacements along the three measurement directions is about $20 \mu \mathrm{m}$. 


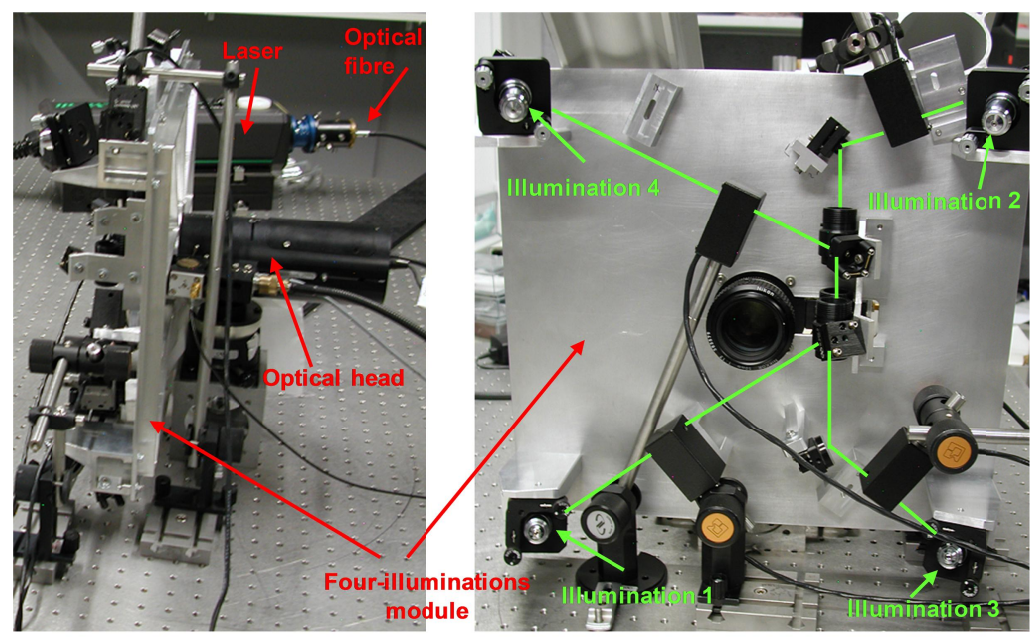

Figure 3. Four-illuminations configuration of the HC

\subsubsection{Validation of the new instrument}

The configuration of the new instrument was imposed by the geometry of a vacuum chamber, it was therefore crucial to validate the performances of the instrument in the lab and above all to determine the measurement uncertainties.

Using the four-illumination holographic technique implies taking great care when implementing the test set-up to limit contributions to the measurement uncertainties. A good mechanical stability between the HC and the tested object is required; both have therefore been implemented on the same optical table. Large objects are tested and the illuminations are not normal to the surface, it is therefore necessary to improve the laser light scattering of the surface by applying white powder on the object. Surfaces with steps generate shadow areas where measurements cannot be performed. Such shadow areas create noise that could affect the phase unwrapping by giving rise to artificial discontinuous areas. Small displacements of the illuminations with respect to the tested object will be interpreted as a displacement of the object. It is therefore important that the four illuminations do not move during the measurement. The four-illuminations module being made of aluminium, the temperature of the $\mathrm{HC}$ must therefore be kept constant during the test to avoid any additional measurement errors. The laser frequency must also be stable during the test to avoid any additional contribution to the measurement uncertainty. A spectrum analyser has therefore been implemented at the output of the laser to check and compensate the frequency drift when necessary.

The post processing of the four phase maps obtained with the $\mathrm{HC}$ involves solving a linear system. This is the crucial step in the post processing: calculate the four phase offsets. Minimizing the noise in the data is therefore a big issue to have reliable results. It was therefore decided to apply a median filter to the phase data to reduce the noise, and to remove the possible outliers. In addition, all noisy areas, e.g. due to shadows of the laser beams or holes, have to be excluded from the post processing. Another point to consider when solving the linear system is the conditioning of the sensitivity matrix in equation (4). This conditioning depends on the variations of the sensitivity vector. For instance for a smaller object observed at the same distance, the matrix conditioning will be worse than for a larger object, the results will therefore be more affected by the noise in the data and the measurement uncertainty will increase. Thus, it is important to have the tested object as close as possible to the HC, this distance being defined by the imaging capabilities of the HC. Finally, the post processing will be more reliable for an object imaged on the whole CCD than for an object partly filling the $\mathrm{CCD}$, as for instance an elongated bar.

The expected measurement uncertainty was $80 \mathrm{~nm}$ for the relative IP and OP displacements since the post processing consists in combining four phase maps. For the absolute displacements, the measurement uncertainty had to be investigated. The system has been validated in the lab first by performing tests on an aluminium plate mounted on a three-axis translation stage to introduce rigid body translations. The amplitude of the translations was measured in parallel with a commercial interferometer (measurement uncertainty $10 \mathrm{~nm}$ ) to estimate the measurement uncertainty of the absolute IP and OP displacements. Then thermal tests have been performed on a $60 \mathrm{~mm}$ Silicon Carbide (SiC) disc 
with a well-known CTE to check the measurement of the relative IP displacement. Some of these tests were performed with non-optimized conditioning of the sensitivity matrix, which significantly degraded the $\mathrm{HC}$ results compared to the expected results. The implemented solution to check the reliability of the measurements and to apply a correction to the measurement if needed was to add a reference sample in the field of view of the HC. For this solution to work properly, the phase difference between the surface of the reference sample and the one of the tested object has to be lower than $2 \pi$ to avoid phase ambiguities. For the test campaigns, this reference was a sample of Zerodur with a length of $70 \mathrm{~mm}$ and a $\mathrm{CTE}$ of $0.05 \times 10^{-6} / \mathrm{K}$. It was located away from the heated area and its temperature variation was less than $2^{\circ} \mathrm{C}$ for all the tests; it could therefore be considered to have zero expansion compared to the measurement uncertainties involved. Also, the same test is performed at least three times to check the consistency and repeatability of the results.

The measurement uncertainties obtained from this validation are:

- $\quad \pm 120 \mathrm{~nm}$ for the relative displacements

- $\quad \pm 230 \mathrm{~nm}$ for the absolute in-plane displacements

- $\quad \pm 400 \mathrm{~nm}$ for the absolute out-of-plane displacements

\section{TESTING}

\subsection{First phase: testing of CFRP samples}

In the first phase of the study two different types of samples have been tested with the HC in a two-illuminations configuration. The tests performed on $\mathrm{SiC}$ samples have been presented in ${ }^{[6]}$. In this paper, the tests performed on Carbon Fiber Reinforced Plastic (CFRP) struts with glued titanium end-fittings are presented. These samples are representative of the connections/junctions used in stable structures and are identified as significant contributors to the instrument stability.

The geometry of the three samples is presented in Figure 4. A fourth CFRP strut without end-fittings has also been tested. The physical properties of the materials involved are assumed to be well-known.
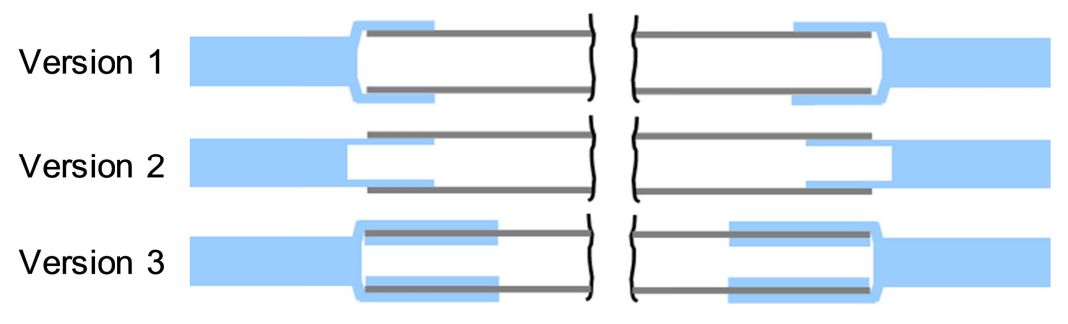

Figure 4. Description of the three types of bonding between the CFRP struts and the titanium end-fittings

\subsubsection{Test set-up}

The four struts are located in a vacuum chamber with an inner diameter of $500 \mathrm{~mm}$ and a height of $545 \mathrm{~mm}$. The primary pump used allows reaching a vacuum of about $10^{-2}$ mbar. The struts lie on two Invar supports located at their extremities and these supports are clamped to an Invar bench mounted on three Invar blades screwed to the bottom flange of the chamber. The temperature variations of the samples are obtained by circulating a mixture of glycol and water in a shroud located underneath the Invar bench. The temperature of the bench can be regulated by a chiller from $-28^{\circ} \mathrm{C}$ to $60^{\circ} \mathrm{C}$. To decrease the temperature gradient in the struts, black painted copper plates have been implemented along each side of the struts. The samples are equipped with thermocouples (TCs) and are coated with white powder (Figure 5a).

The support of the HC is an assembly of columns to make it as stable and rigid as possible. The whole set-up is placed on an optical table (Figure 5b). 


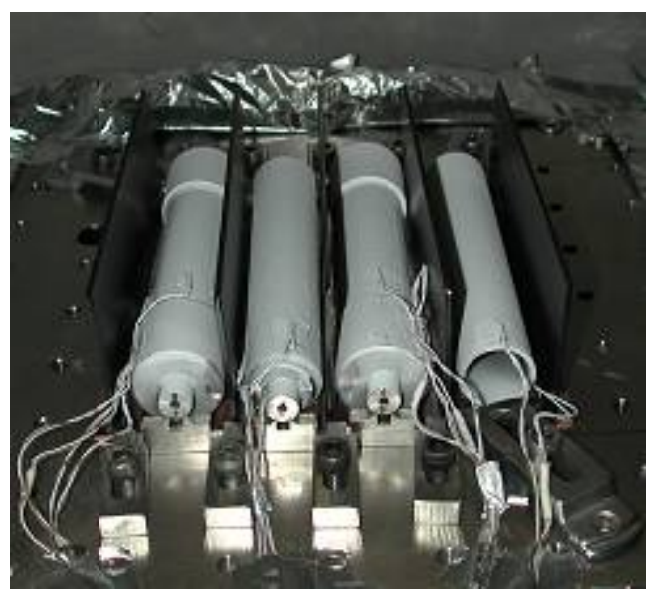

(a)

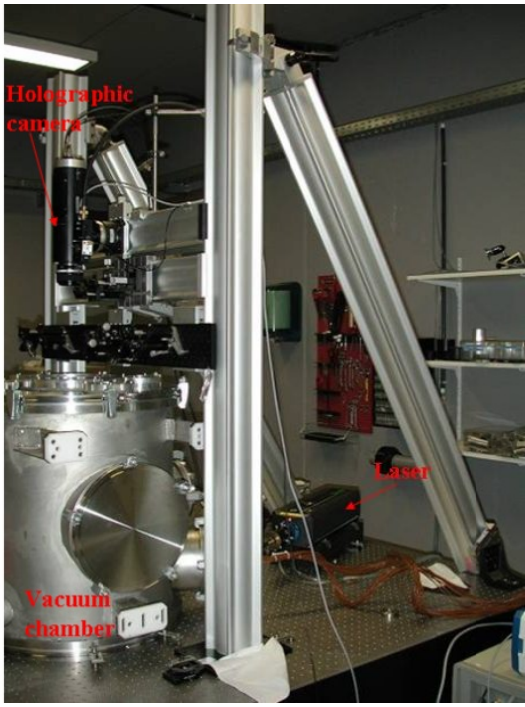

(b)

Figure 5. (a) Integration of the CFRP struts in the vacuum chamber and (b) Test set-up

\subsubsection{Test results}

The success criteria of the tests are defined as follows:

- Homogeneous temperature on samples achieved for each acquisition: less than $0.5 \mathrm{~K}$ variations in the sample (in plane and in the height of the sample).

- Comparison with reference case after heating or cool down shows no samples variations.

- Shape of the computed displacements is comparable with the predictions. The differences between the displacements measured and the predictions are within $+/-25 \%$.

The criterion for the temperature stabilisation was to have less than $0.1^{\circ} \mathrm{C}$ variation over 10 minutes for each $\mathrm{TC}$ (relative measurement uncertainty of $0.1 \mathrm{~K}$ ).

Tests have been performed for temperature variations between $-10 \mathrm{~K}$ and $+15 \mathrm{~K}$ from room temperature (RT). An example of phase images obtained with the $\mathrm{HC}$ is presented in Figure 6. The post processing of the results consisted in scaling the data for each sample by considering the CFRP CTE and the strut expansion in the center area to avoid the influence of bi-metallic effects near the end-fittings. Also, the tilts in the out-of-plane relative displacement results, caused by the rigid body movements of the samples, were removed by computing the Zernike coefficients.

An example of OP and IP displacements measured for the hot case $\mathrm{RT}+15 \mathrm{~K}$ is presented in Figure 7 . The noisy areas are due to the stickers of the TCs and the shadow due to the geometry of the struts that prevents the unwrapping algorithm from performing well in these areas. The success criteria have been matched for all the samples.
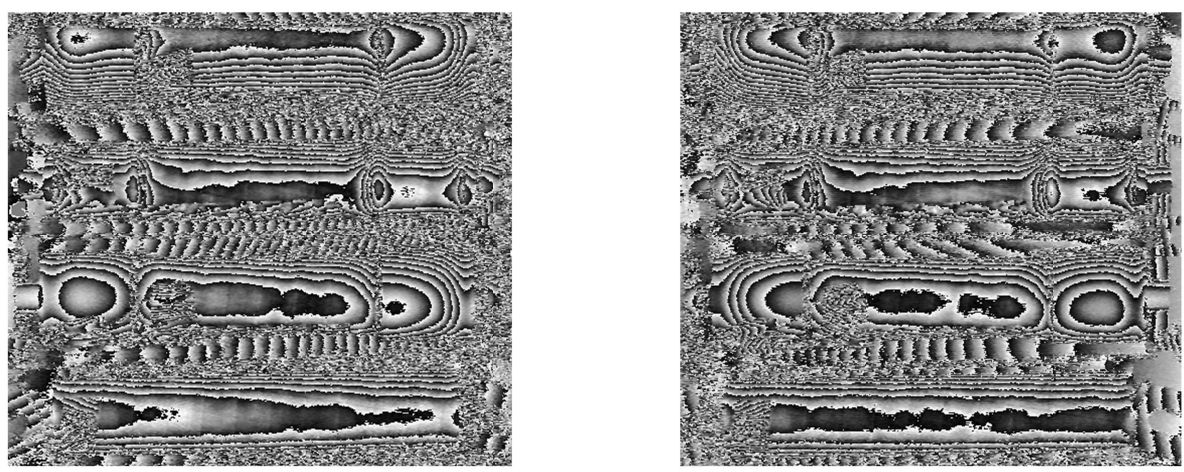

Figure 6. Phase images for the hot case $\mathrm{RT}+15^{\circ} \mathrm{C}$. 


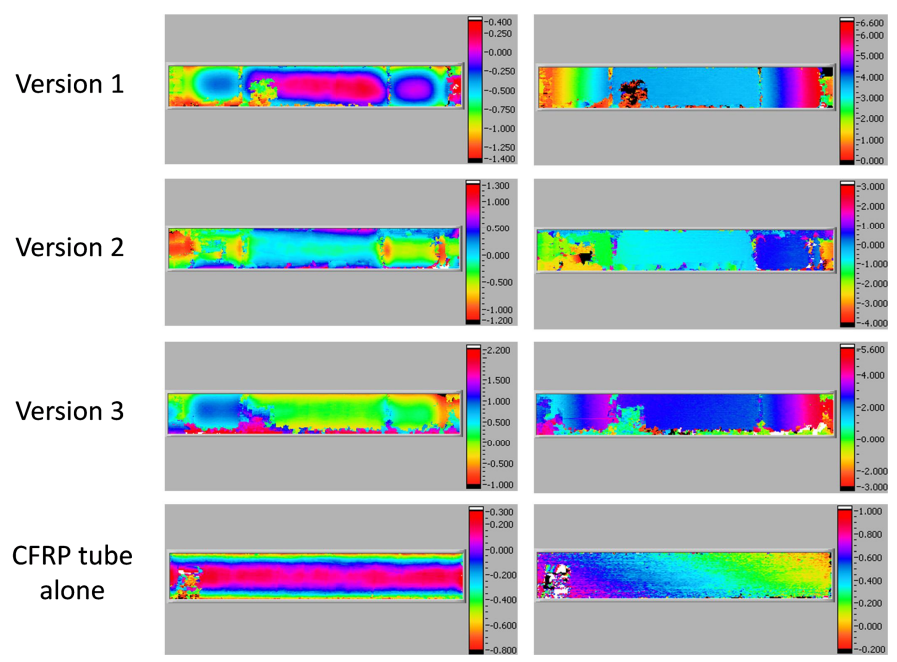

Figure 7. OP (left) and IP (right) displacements measured for the hot case RT $+15^{\circ} \mathrm{C}$.

\subsection{Second phase: testing of representative flight hardware}

The second phase of the test campaign was performed on a flight representative instrument structure with the HC in a four-illuminations configuration. Two different types of structure have been tested: a SiC structure and a composite structure with GFRP struts and Titanium end-fittings. The results obtained on the full $\mathrm{SiC}$ structure have been presented in ${ }^{[9]}$. In the current paper, tests performed on a structure composed of a large SiC base plate supported by three Glass Fiber Reinforced Plastic (GFRP) struts with glued titanium end-fittings (Figure 8) are presented. This assembly is representative of stable structure for space applications. The physical properties of the materials involved are assumed to be well-known. The three struts are equipped with a heater glued on the central part of the struts. Two areas of interest (in red in Figure 8) have been measured by the HC.

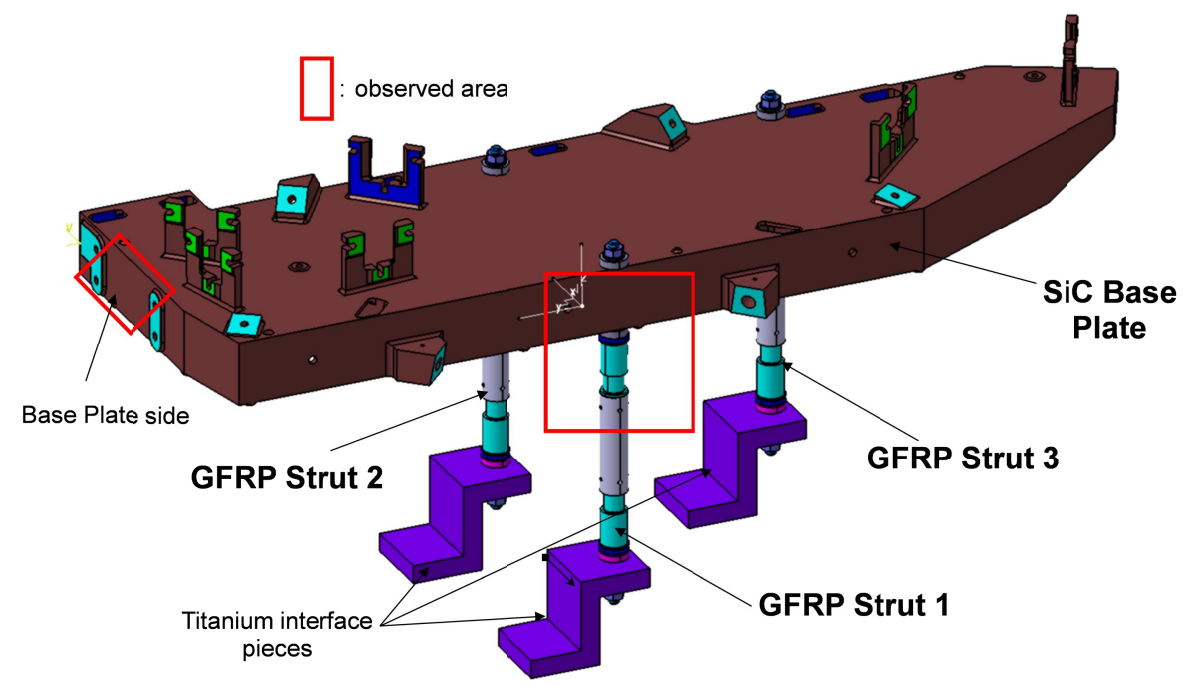

Figure 8. Structure composed of a $\mathrm{SiC}$ base plate supported by 3 GFRP struts.

\subsubsection{Test set-up}

The test set-up for measuring the displacement of the strut and the $\mathrm{SiC}$ base plate is presented in Figure 9a. The structure is clamped to an optical table. The area to be measured is coated with white powder. The Zerodur sample is put on the base plate. TCs are glued on the struts and the SiC base plate. A thermographic camera (not shown in Figure 9a) observing the $\mathrm{SiC}$ base plate is also used to check the temperature gradient in the base plate. Since a stable temperature 
of the HC is needed, the test set-up is implemented in a closed room and is controlled from outside. The measurements are performed once the thermal equilibrium is reached in the room. The temperature of the measurement system is monitored by TCs. The area of interest measured by the HC for this test set-up is presented in Figure 9b.

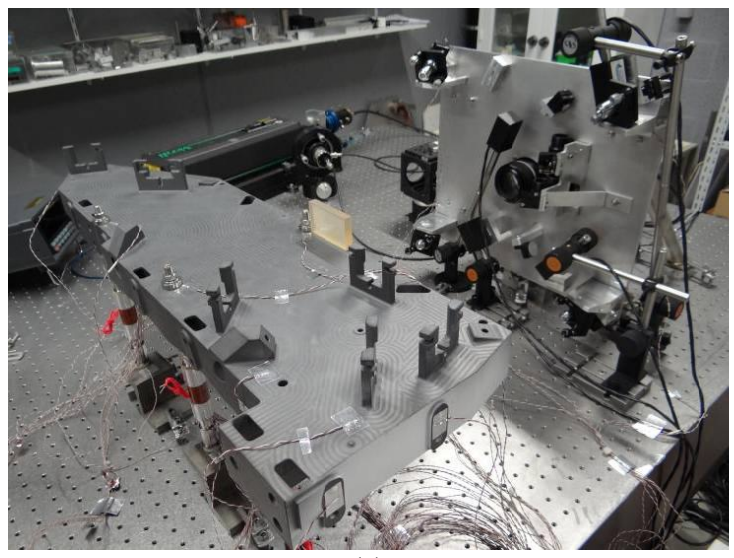

(a)

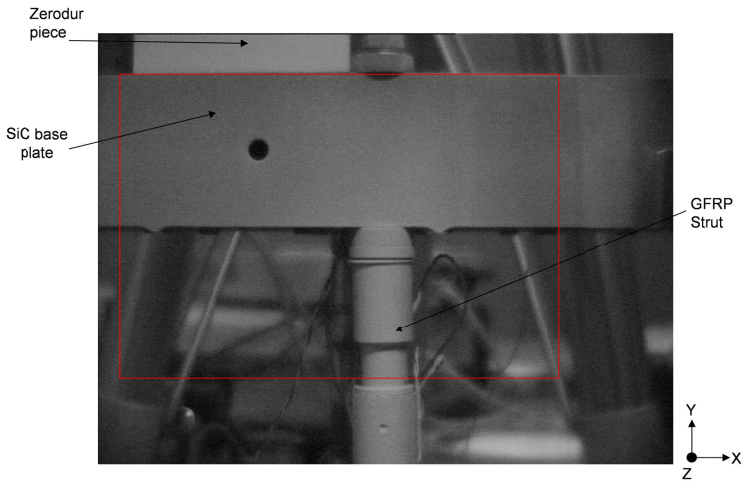

(b)

Figure 9. (a) Test set-up and (b) FOV of the HC for this test set-up.

\subsubsection{Test results}

The success criteria of the tests are defined as follows:

- Temperature variation at the $\mathrm{HC}$ level is $\leq 0.01 \mathrm{~K}$ (temperature variation is filtered using a moving average).

- The measurement of the expected expansion of the Zerodur sample is within the measurement uncertainty, with a scaling of the results if necessary.

- For each load case at least 2 tests in the same configuration will be performed; the difference between the test results will be within the measurement uncertainties, taking into account the possible difference in the temperature distribution.

- Shape of the measured displacements is comparable with the initial predictions.

- Amplitude of the measured displacements are:

- Of the same order of magnitude as the one in the initial predictions.

$\circ$ Or the difference with predictions is within the measurement uncertainties if this difference is higher than $25 \%$ of the predictions.

Some tests have been performed by heating one, two or the three struts. The heating lasts only a few minutes to create temperature gradients in the connections. The temperature variations at the heater level are typically between $18 \mathrm{~K}$ and $30 \mathrm{~K}$.

An example of phase images obtained with the $\mathrm{HC}$ is presented in Figure 10a. Post processing of these data produced the three displacement maps in Figure 10b. For this test, no scaling of the results to match the Zerodur expected expansion was needed. 

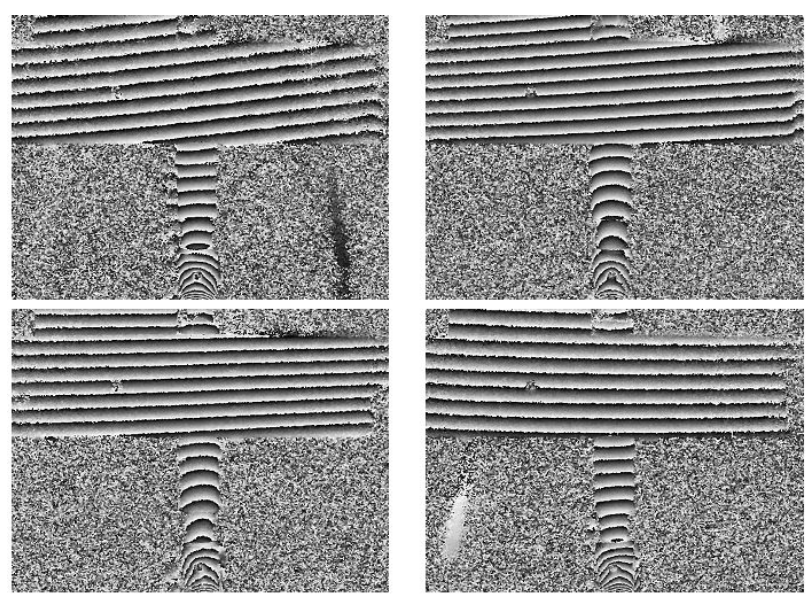

(a)
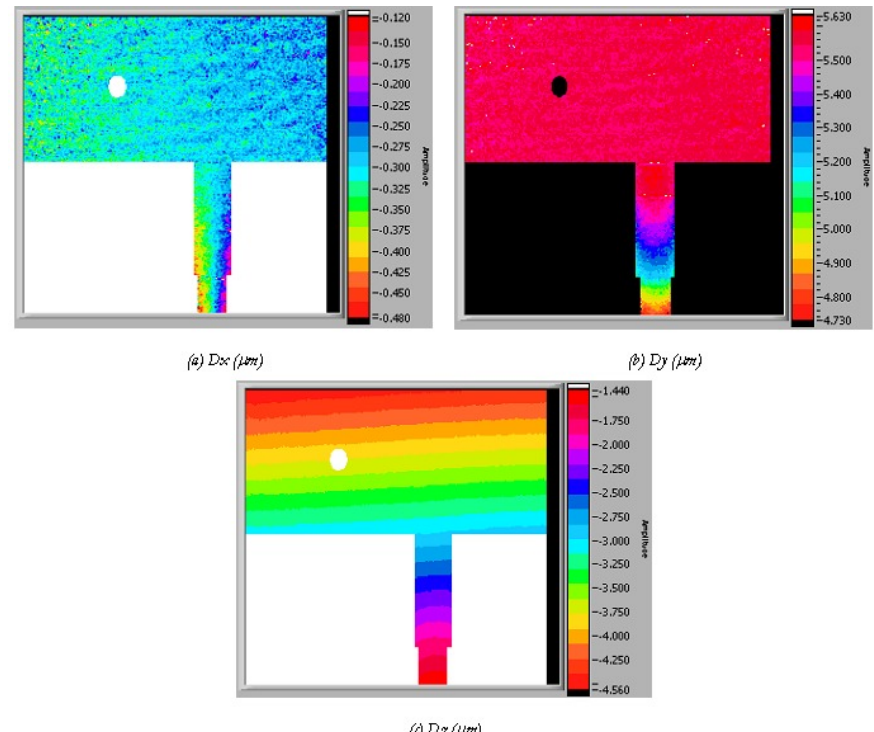

(b)

Figure 10. (a) Phase images obtained with the HC and (b) the 3 components of displacement obtained from these phase images

\section{COMPARISON TESTS/SIMULATIONS}

The test results of the first phase of the study have been compared to the Finite Element Modeling (FEM) predictions performed by EADS Astrium. The objective was to improve and develop analytical predictions and verification of thermo-elastic distortions, assuming that the physical properties of the materials involved were well-known. For the relative IP and OP displacement a good correlation between test and prediction results has been obtained for all versions of the struts and all load cases performed. Thus the FEM delivers good results, in particular for the axial displacements, which is the most important property for strut analysis as it is the axial displacement that will impact the overall stability performances of a structure involving struts. For those composite samples, the test results confirmed that the FEM results are reliable.

For the second phase of the study on the flight representative hardware, a FEM has been built by EADS Astrium with respect to the modeling guidelines issued in phase 1. Using this model, an analysis has been implemented to perform the correlation between the test and prediction results. First a thermal analysis establishes the temperature field. Then with the temperature map as an input, the displacement field of the whole structure is obtained from thermo-elastic distortion analysis. The displacement data is then processed to match with the format of the test result. Finally the computed displacements are compared to the measured ones.

Five different load cases have been studied, an example of comparison between experimental and FEM results is presented in Figure 11. This load case was performed by heating strut 1 (delta temperature about 18K at heater level) and measuring this strut 1 and part of the $\mathrm{SiC}$ base plate, as shown in Figure 9b. The first row of subfigures corresponds to experimental results. The second row corresponds to prediction results. The last row presents a difference, pixel by pixel, between the experimental and numerical data. 

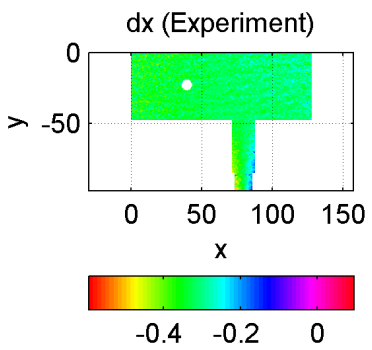

$\mathrm{dx}(\mathrm{FEM})$
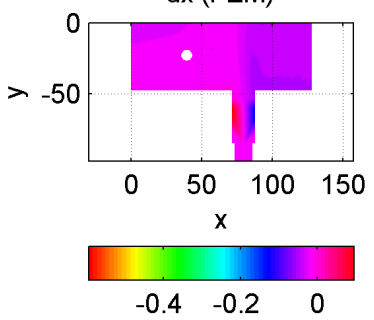

$\mathrm{dx}($ Error in $\mu \mathrm{m})$
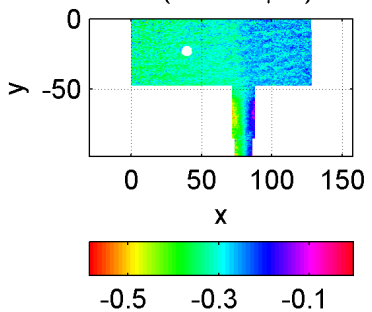

dy (Experiment)
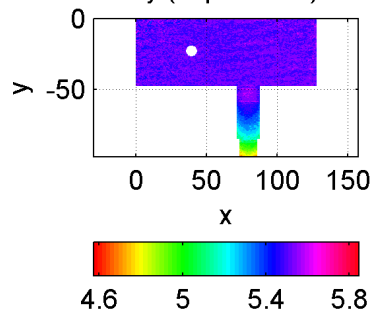

dy (FEM)
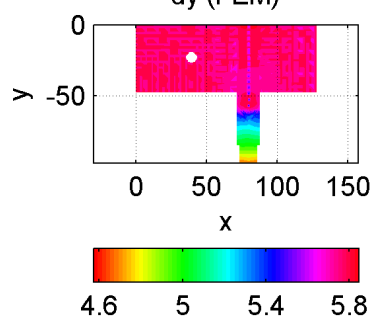

dy (Error in $\mu \mathrm{m})$
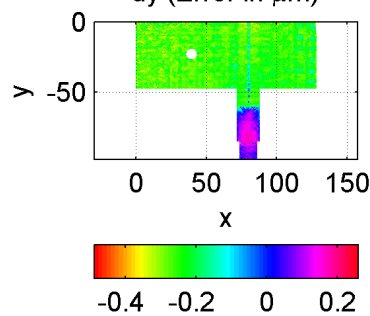
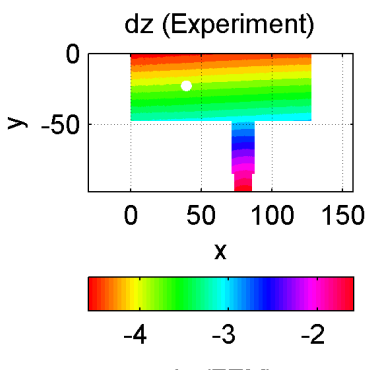

$\mathrm{dz}(\mathrm{FEM})$
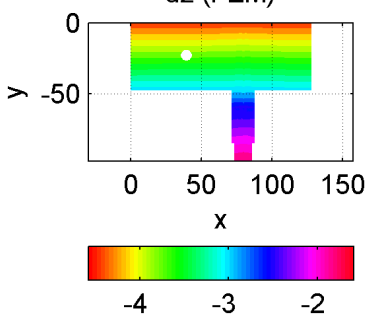

$\mathrm{dz}($ Error in $\mu \mathrm{m})$
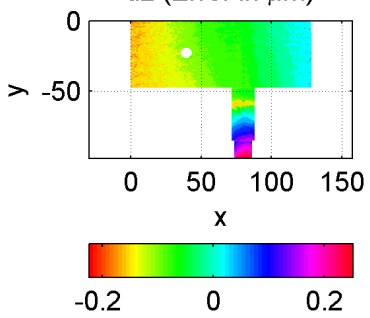

Figure 11. Comparison between prediction and test results (from results in Figure 10)

For the correlation analysis of all the results obtained during this second phase, the global uncertainty includes the major identified uncertainties, except the ones on material properties that are considered as well-known in this study. This includes the uncertainty on the test results from the $\mathrm{HC}$, but also the uncertainty on the temperature map. Table 1 presents the percentage of results within the global uncertainty range and within two times this uncertainty range. This table is a synthesis of the results obtained for the five load cases performed on the structure and not only the one presented as an example. $58 \%$ of the absolute IP displacement results are within the global uncertainty, but axial displacements are $100 \%$ within this uncertainty: the main objective of this second phase, that was to correctly predict the axial displacement of the struts that have a dominant effect on structure stability, is reached.

Table 1. Fractions of five load cases results within global uncertainty range or within two times this uncertainty

\begin{tabular}{|c|l|c|c|}
\cline { 3 - 4 } \multicolumn{1}{|c|}{} & \multicolumn{2}{|c|}{ Unce rtainty } \\
\cline { 3 - 4 } Abs olute & in range & in 2x range \\
\hline & in-plane global & $60 \%$ & $80 \%$ \\
\cline { 2 - 4 } & in-plane Dy & $\mathbf{1 0 0 \%}$ & $\mathbf{1 0 0 \%}$ \\
\cline { 2 - 4 } & out-of-plane & $80 \%$ & $100 \%$ \\
\cline { 2 - 4 } Relative & overall & $67 \%$ & $87 \%$ \\
\hline \multirow{4}{*}{ Combine d } & in-plane global & $80 \%$ & $100 \%$ \\
\cline { 2 - 4 } & in-plane Dy & $\mathbf{6 0 \%}$ & $\mathbf{1 0 0 \%}$ \\
\cline { 2 - 4 } & out-of-plane & $60 \%$ & $100 \%$ \\
\cline { 2 - 4 } & overall & $73 \%$ & $100 \%$ \\
\hline & in-plane global & $70 \%$ & $90 \%$ \\
\cline { 2 - 4 } & out-of-plane & $\mathbf{8 0 \%}$ & $\mathbf{1 0 0 \%}$ \\
\hline & overall & $70 \%$ & $100 \%$ \\
\hline
\end{tabular}




\section{CONCLUSIONS}

Different configurations of a photorefractive $\mathrm{HC}$ have been presented. The two-illuminations configuration showed limitations that have been overcome by upgrading the instrument to a four-illuminations configuration to measure the three components of displacements. This instrument has been validated in the lab and the measurement uncertainties have been estimated. The implementation, performances and limitations of this instrument have been presented.

These two configurations have been used during an ESA study on thermo-elastic distortion verification methods. This paper has presented some results obtained on composite structure in CFRP and GFRP. Prediction and test results have been compared: the strut axial displacement, which has been identified as the main contributor to overall thermo-elastic stability of a structure equipped with struts, was well correlated. This type of measurement has also been successfully performed on $\mathrm{SiC}$ structures with bolted and glued assemblies ${ }^{[6],[9]}$.

The $\mathrm{HC}$ has evolved from a one-illumination configuration to a four-illuminations configuration that allows measuring the three components of the displacement on a whole field $\left(250 \times 250 \mathrm{~mm}^{2}\right)$ and without contact. The technique can still be improved, for instance by making the post processing more robust to get rid of the reference in the FOV.

\section{REFERENCES}

[1] Georges, M.P., Scauflaire, V. and Lemaire, P.C, "Compact and portable holographic camera using photorefractive crystals. Applications in various metrological problems," Appl. Phys. B 72, 761-765 (2001).

[2] Lemaire, P.C. and Georges, M.P., "Dynamic holographic interferometry: devices and applications," in [Photorefractive Materials and Their Applications 3], Günter, P., and Huignard, J.P., eds, Springer Series in Optical Sciences Vol 11, p.223-251 (2007).

[3] Georges, M.P. and Lemaire, P.C., "Phase-shifting real-time holographic interferometry that uses bismuth silicon oxide crystals," Applied Optics 34, 7497-7506 (1995).

[4] Georges, M.P. and Lemaire, P.C., "Real-time holographic interferometry using sillenite photorefractive crystals. Study and optimization of a transportable set-up for quantified phase measurements on large objects," Appl. Phys. B 68, 1073-1083 (1999).

[5] Thizy, C., Georges, M.P., Scauflaire, V., Lemaire, P.C. and Ryhon,S., "In-plane and out-of-plane holographic interferometry with multibeam photorefractive recordings in sillenite photorefractive crystals," Trends in Optics and Photonics Series, Vol 87 on Photorefractive Effects, Materials and Devices, 504-510 (2003).

[6] Eliot, F., Thizy, C., Shannon, A., Stockman, Y., Logut, D., "Thermo-elastic distortion measurements by holographic interferometry and correlation with finite element models for $\mathrm{SiC}$ connections/junctions on spacecraft," Proceedings of the 61st International Astronautical Congress, 1993-2006 (2010).

[7] Stetson, K.A., "Use of sensitivity vector variations to determine absolute displacements in double exposure hologram interferometry," Applied Optics 29, 502-504 (1990).

[8] Thizy, C., Georges, M.P., Scauflaire, V. and Lemaire, P.C., "Study and comparison of multibeam photorefractive recordings and photorefractive crystals stack to holographic interferometry," Trends in Optics and Photonics Series, Vol 87 on Photorefractive Effects, Materials and Devices, 469-475 (2003).

[9] Eliot, F., Thizy, C., Ballhause, D., Laduree, G., Logut, D., "3D thermo-elastic distortion measurements on OSIRIS $\mathrm{SiC}$ hardware by holographic interferometry and correlation with finite element models," Proceeding of the $12^{\text {th }}$ European Conference on Spacecraft Strucutres, Materials \& Environmental testing (2012). 